\title{
Scaling of high-energy elastic scattering and the observation of Odderon
}

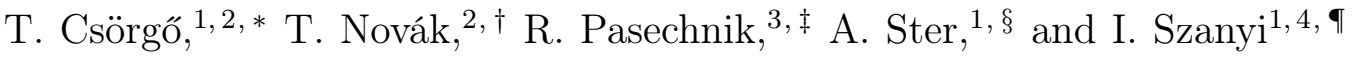 \\ ${ }^{1}$ Wigner RCP, H-1525 Budapest 114, POBox 49, Hungary \\ ${ }^{2}$ SZIE KRC, H-3200 Gyöngyös, Mátrai út 36, Hungary \\ ${ }^{3}$ Department of Astronomy and Theoretical Physics, \\ Lund University, 22100 Lund, Sweden \\ ${ }_{4}^{4}$ Eötvös University, H-1117 Budapest, Pázmány P. s. 1/A, Hungary
}

\begin{abstract}
We provide a statistically significant observation of the elusive Odderon exchange, based on novel and model-independent analysis of the scaling properties of the differential cross sections of elastic $p p$ and $p \bar{p}$ scattering in the $\mathrm{TeV}$ energy range. We report the statistical significance of the observed Odderon signal at the level of $6.26 \sigma$.
\end{abstract}


In 1973, Lukaszuk and Nicolescu [1] proposed that a noticeable crossing-odd contribution called Odderon may be present in the scattering amplitude of elastic proton-proton $(p p)$ and proton-antiproton $(p \bar{p})$ scattering at asymptotically high energies. In the field theory of strong interactions, quantum chromodynamics (QCD), the Odderon exchange corresponds to the $t$-channel exchange of a color-neutral gluonic compound state consisting of an odd number of gluons, as elaborated by Bartels, Lipatov and Vacca in Ref. [2]. Although more than 20 years have passed since the theoretical prediction of the Odderon in QCD, and over 46 years since the Odderon concept has been introduced in Regge phenomenology, the Odderon remained elusive so far due to lack of a definitive experimental evidence. A direct way to probe the Odderon in elastic scattering is by comparing the differential crosssection of particle-particle and particle-antiparticle scattering at the same and sufficiently high energy [3, 4]. The first search performed at the ISR energy of $\sqrt{s}=53 \mathrm{GeV}$ in 1985 [5] resulted in an indication of the Odderon at the $3.35 \sigma$ significance level. That analysis, however, did not utilize all the available data in the overlapping acceptance of the $p p$ and $p \bar{p}$ measurements. Furthermore, at such a low energy the Reggeon exchanges are expected to play a significant role rendering the Odderon search at the ISR rather inconclusive.

Recently, the TOTEM Collaboration published a series of important papers investigating the properties of elastic $p p$ scattering in the LHC energy range between $\sqrt{s}=2.76$ and 13 $\mathrm{TeV}$ [6 9]. An increase of the total cross section, $\sigma_{\text {tot }}(s)$, associated with a decrease of the real-to-imaginary ratio, $\rho(s)$, with energy, first identified at $\sqrt{s}=13 \mathrm{TeV}$ [6, 7] indicated a possible Odderon effect triggering an intense debate [10 27]. The persistent diffractive minimum-maximum structure in the $t$-dependent profile of $d \sigma / d t$ in elastic $p p$ collisions observed by the TOTEM at $\sqrt{s}=2.76,7$ and $13 \mathrm{TeV}$, and the lack of such structure in elastic $p \bar{p}$ collisions measured by D0 [28] at $\sqrt{s}=1.96 \mathrm{TeV}$, indicate a qualitatively clear Odderon effect [12]. Thus the TOTEM collaboration concluded in Ref. [9] as follows: "Under the condition that the effects due to the energy difference between TOTEM and DO can be neglected, the result provides evidence for a colourless 3-gluon bound state exchange in the t-channel of the pp elastic scattering". However, no conclusive, quantitative experimental results were published so far with a statistically significant evidence for an Odderon discovery.

In this work, we present a definitive and statistically significant Odderon observation. This result is based on a re-analysis of already published D0 [28] and TOTEM [8, 9, 29] data sets, without the use of any fitting function or theoretical input. Namely, we compare 
pairwise the scaling functions constructed at different energies based upon the available data and look for statistically significant differences within any pair of TeV-scale $p p$ and $p \bar{p}$ data sets depending on the collision energy.
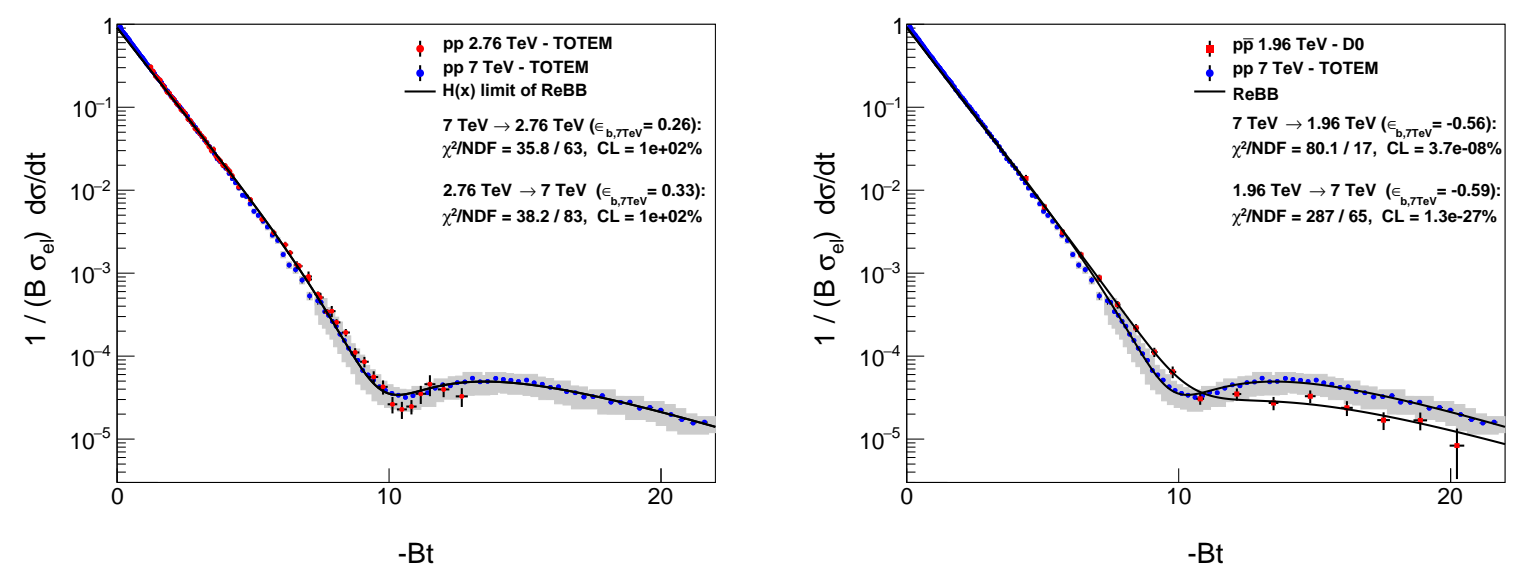

FIG. 1: Left panel indicates that for $p p$ elastic scattering the $H(x)$ scaling function for $x=-t B$ is energy independent in the energy range of $\sqrt{s}=2.76-7 \mathrm{TeV}$. Vertical and horizontal lines on each point stand for the corresponding type A errors. Grey vertical bars represent the type B (vertical and horizontal) errors. The right panel indicates a statistically significant difference between the $H(x)$ scaling functions for elastic $p p$ collisions at $\sqrt{s}=7 \mathrm{TeV}$ and that of $p \bar{p}$ collisions at $\sqrt{s}=1.96$ $\mathrm{TeV}$ at the level of at least $6.26 \sigma$. Here, $X \rightarrow Y$ denotes projections by exponential interpolation between the two adjacent data points of the data set $X$ to get its $H(x)$ at the same $x$ as that of the other data set $Y$ to be able to compare them via $\chi^{2}$-method as described in the text. On the left panel, solid line indicates the $H(x)$ scaling limit of the ReBB model 3032]. On the right panel, solid line indicates the full ReBB model result, including scaling violations [30 32]. Both solid lines correspond to Refs. [31, 32].

Our analysis is based on a novel scaling law of elastic $p p(p \bar{p})$ scattering [33]. We utilize a new kind of scaling function, $H(x, s)=\left(1 / B \sigma_{\mathrm{el}}\right) d \sigma / d t$, where $x=-t B$, and

$$
\sigma_{\mathrm{el}}=\int_{0}^{\infty} d|t| \frac{d \sigma}{d t}, \quad B=\left.\frac{d}{d t} \ln \frac{d \sigma}{d t}\right|_{t \rightarrow 0},
$$

that has been found to be energy independent, $H\left(x, s_{1}\right)=H\left(x, s_{2}\right)$, in elastic $p p$ collisions in a few TeV scattering domain, within the acceptance of TOTEM measurements at 2.76, 7 (and 8) $\mathrm{TeV}$ as demonstrated in the left panel of Fig. 1. This $H(x)$ scaling removes the trivial energy dependent terms, due to the known $s$-dependence of the elastic slope $B(s)$, the elastic 
and total cross-sections $\sigma_{\mathrm{el}}(s)$ and $\sigma_{\text {tot }}(s)$, and the real-to-imaginary ratio $\rho(s)$ [34]. For $p p$ elastic scattering the $H(x, s)$ scaling function for $x=-t B$ is energy independent in the energy range of $\sqrt{s}=2.76-7 \mathrm{TeV}$, as shown on the left panel of Fig. 11 within statistical errors, the agreement corresponds to the confidence level (CL) of $99 \%$. Combining both the statistical and systematic errors, the agreement corresponds to a $\chi^{2} / \mathrm{NDF}=35.8 / 63$ in $1.2<x<12.7$, with a $\mathrm{CL}=100 \%$. A quantitative Odderon effect is then determined by a projection of the $p p$ and $p \bar{p}$ data and the corresponding uncertainties to the same $x(s)$ values at different energies. The $7 \rightarrow 1.96 \mathrm{TeV}$ and $1.96 \rightarrow 7 \mathrm{TeV}$ projections correspond to keeping the measured $x$ values at $\sqrt{s}=1.96$ and $7 \mathrm{TeV}$, respectively, and determining by interpolation the $H(x, s)$ scaling functions at these $x$ values, but at the other energy, $\sqrt{s}=7$ and $1.96 \mathrm{TeV}$, respectively. The right panel of Fig. 1 compares the $H(x)$ scaling function of elastic $p p$ collisions at $\sqrt{s}=7 \mathrm{TeV}$ to that of $p \bar{p}$ collisions at $\sqrt{s}=1.96 \mathrm{TeV}$. In this case, adopting the method of Ref. [35], the confidence level of the agreement of the $H(x)$ scaling functions is found to be maximum $3.7 \times 10^{-8 \%}$, with a minimum of $\chi^{2} / \mathrm{NDF}=80.1 / 17$. Hence, the difference between these scaling functions is statistically significant and represents our main result for the Odderon observation as at least $6.26 \sigma$ effect, with probability, $P=1-\mathrm{CL}=0.999999963$, in the $5<x \lesssim 20$ acceptance. This is a conservative result as we find that this value is robust (can only increase) for the variation of the procedure and the $\chi^{2}$ definition. To guide the eye, solid lines, representing the ReBB model results [30 32] are also added to both panels of Fig. 1.

As a cross-check, we have tested the validity of the $H(x)$ scaling versus the TOTEM data on elastic $p p$ scattering at $\sqrt{s_{1}}=7 \mathrm{TeV}$ and TOTEM preliminary data and errors at $\sqrt{s_{2}}=8$ $\mathrm{TeV}$ [36]. We find that $H\left(x, s_{1}\right)=H\left(x, s_{2}\right)$ at $\mathrm{CL} \approx 100 \%$ in the $5 \leq x \leq 20$-range. Due to a lack of direct measurements of $p p$ and $p \bar{p}$ collisions at exactly the same energy in the $\mathrm{TeV}$ region, we utilize the energy independence of the $H(x)$ scaling in the LHC energy range of $1 \lesssim \sqrt{s} \lesssim 8 \mathrm{TeV}$, to evaluate the characteristics of the elastic $p p$ scattering at the D0 energy of $\sqrt{s}=1.96 \mathrm{TeV}$.

We have also cross-checked this scaling behaviour at ISR energies and found that all the differential cross sections of elastic $p p$ scattering, measured at the ISR energy range of $\sqrt{s}=$ $23.5-62.5 \mathrm{GeV}$ [37, 38], can approximately be scaled to the same universal curve [33]. We have also studied the $H(x)$ scaling for elastic $p \bar{p}$ collisions in the energy range of $\sqrt{s}=0.546-$ $1.96 \mathrm{TeV}$ and found that in this case, the scaling is limited to the diffractive cone, $x \leq 10$ only, 
where $H(x) \approx \exp (-x)$, but in $p \bar{p}$ collisions the $H(x)$ scaling is strongly and qualitatively violated for $x>10$ values. However, the valid $H(x)$ scaling in $p p$ scattering allowed us to scale down the higher energy TOTEM $p p$ data from $\sqrt{s}=7 \mathrm{TeV}$ to $\sqrt{s}=1.96 \mathrm{TeV}$ and directly compare it to the D0 $p \bar{p}$ data.

The quantification of the Odderon significance is based on a method developed by the PHENIX collaboration in Ref. [35] using a specific $\chi^{2}$ definition that effectively diagonalizes the covariance matrix. In the PHENIX formulation, the experimental data are compared to a theoretical calculation. In our analysis, we adapt the PHENIX method for comparison of one set of data directly to another set of data, without using any theory or fitting functions. Following the PHENIX method, we classify the experimental errors of a given data set into three different types: (i) type A, point-to-point fluctuating (uncorrelated) systematic and statistical errors, (ii) type B errors that are point-to-point dependent, but $100 \%$ correlated systematic errors, and (iii) type $\mathrm{C}$ errors, that are point-to-point independent, but fully correlated systematic errors [35] to evaluate the significance of correlated data, when the covariance matrix is not publicly available. Since the $t$-dependent systematic errors in TOTEM measurements are almost $100 \%$ correlated, we classified them as type B errors, while the $t$-independent overall normalization errors are type $\mathrm{C}$ errors, and the statistical errors are type A errors.

The source of the TOTEM $p p$ differential cross section data, measured at $\sqrt{s}=7 \mathrm{TeV}$, is Ref. [29]. In addition, the values of $|t|$ were determined together with their errors of type A and $\mathrm{B}$ as given in Table 5 of Ref. [39] and Table 3 of Ref. [40]. The $t$-independent, type-C errors cancel from the $H(x)$ scaling functions, as they multiply both the numerator and the denominator of $H(x)$. At $\sqrt{s}=2.76 \mathrm{TeV}$, in Ref. [9], the TOTEM Collaboration published the $p p$ differential cross section data with separated type-A and type-B errors. However, the D0 collaboration did not publish type-B errors for its differential cross-section data at $\sqrt{s}=1.96 \mathrm{TeV}[28]$. We have thus fixed the correlation coefficient of these D0 type-B errors to zero. The input values of the nuclear slope parameters $B$ and the elastic cross sections $\sigma_{\text {el }}$ are summarized in Table $\mathbb{I}$, together with the appropriate references.

We define the significance of the agreement between the data set $D_{1}$ and the projection $D_{21}=D_{2} \rightarrow D_{1}$ of data set $D_{2}$ to $D_{1}$ in their overlapping acceptance, with the the following 
$\chi^{2}$ definition [33]:

$$
\begin{aligned}
\chi_{2 \rightarrow 1}^{2} & =\sum_{j=1}^{n_{21}} \frac{\left(d_{1}^{j}+\epsilon_{b, 1} e_{B, 1}^{j}-d_{21}^{j}-\epsilon_{b, 21} e_{B, 21}^{j}\right)^{2}}{\left(\tilde{e}_{A, 1}^{j}\right)^{2}+\left(\tilde{e}_{A, 21}^{j}\right)^{2}}+\epsilon_{b, 1}^{2}+\epsilon_{b, 21}^{2}, \\
\tilde{e}_{A, k}^{j} & =e_{A, k}^{j} \frac{d_{k}^{j}+\epsilon_{b, k} e_{B, k}^{j}}{d_{k}^{j}}, \\
e_{M, k}^{j} & =\sqrt{\left(\sigma_{M, k}^{j}\right)^{2}+\left(d_{k}^{\prime, j}\right)^{2}\left(\delta_{M, k}^{j} x\right)^{2}},
\end{aligned}
$$

where $n_{21}$ is the number of data points $d_{21}^{j}$ in $D_{21}$ indexed by $j$, the same as in $D_{1}$ but remaining in the overlapping acceptance of $D_{1,2}$ sets, $e_{M, k}^{j}, k=1,21$, are the type $M=A, B$ errors found in terms of the type-M vertical errors on data point $j, \sigma_{M, k}^{j}$, added in quadrature with the corresponding type-M vertical errors that were evaluated from the corresponding errors on the horizontal axis $x$ with the scaled variance method, $d_{k}^{\prime, j} \delta_{M, k}^{j} x$, where $d_{k}^{\prime, j}$ stands for the numerical derivative of the measured quantity in data set $D_{k}$ at the point $j$ in the

\begin{tabular}{|c|c|c|}
\hline$\sqrt{s}(\mathrm{GeV})$ & $\sigma_{\mathrm{el}}(\mathrm{mb})$ & $B\left(\mathrm{GeV}^{-2}\right)$ \\
\hline $1960(p \bar{p})$ & $20.2 \pm 1.7^{A} \pm 14.4 \%^{C}\left[{ }^{*}\right]$ & $16.86 \pm 0.1^{A} \pm 0.2^{A}$ \\
\hline $2760(p p)$ & $21.8 \pm 1.4^{A} \pm 6.0 \%^{C} \quad$,9, 41] & $17.1 \pm 0.3^{A}$ \\
\hline $7000(p p)$ & $25.43 \pm 0.03^{A} \pm 0.1^{B} \pm 0.31^{C} \pm 1.02^{C}$ & $19.89 \pm 0.03^{A} \pm 0.27^{B}$ \\
\hline
\end{tabular}
common acceptance and $\delta_{M, k}^{j} x$ is the $j$-dependent type-M horizontal error. The overall correlation coefficients of the type B errors $e_{B, k}^{j}$ of $D_{k}$ data sets are denoted by $\epsilon_{b, k}$.

TABLE I: Summary table of the elastic cross-sections $\sigma_{\mathrm{el}}$ and the nuclear slope parameters $B$, with references. We have indexed with superscripts $A, B, C$ the type A,B,C errors, respectively. The value and the type A error of the elastic cross-section $\sigma_{\mathrm{el}}$ at $\sqrt{s}=1.96 \mathrm{TeV}\left[{ }^{*}\right]$ is obtained from a low $-t$ exponential fit to the data of Ref. [28], while the type C error is from Ref. [28]. The statistical and systematic errors of $d \sigma / d t$ data at $\sqrt{s}=1.96 \mathrm{TeV}$ were added in quadrature in Ref. [28], therefore it was done in case of the elastic slope $B$ as well, providing a combined type A error $\delta^{A} B=0.224$ $\mathrm{GeV}^{-2}$. At $\sqrt{s}=2.76 \mathrm{TeV}$, Ref. [9] provides the total error on $B$, without decomposing it into type A and type B parts. Similarly, the error on the TOTEM preliminary value of the elastic cross section at $\sqrt{s}=2.76 \mathrm{TeV}$ was not decomposed to type $\mathrm{A}$ and $\mathrm{B}$ errors in Ref. [41], either. Hence, we treat these as errors of type A: this assumption yields a conservative estimate of the Odderon significance in our calculations. 

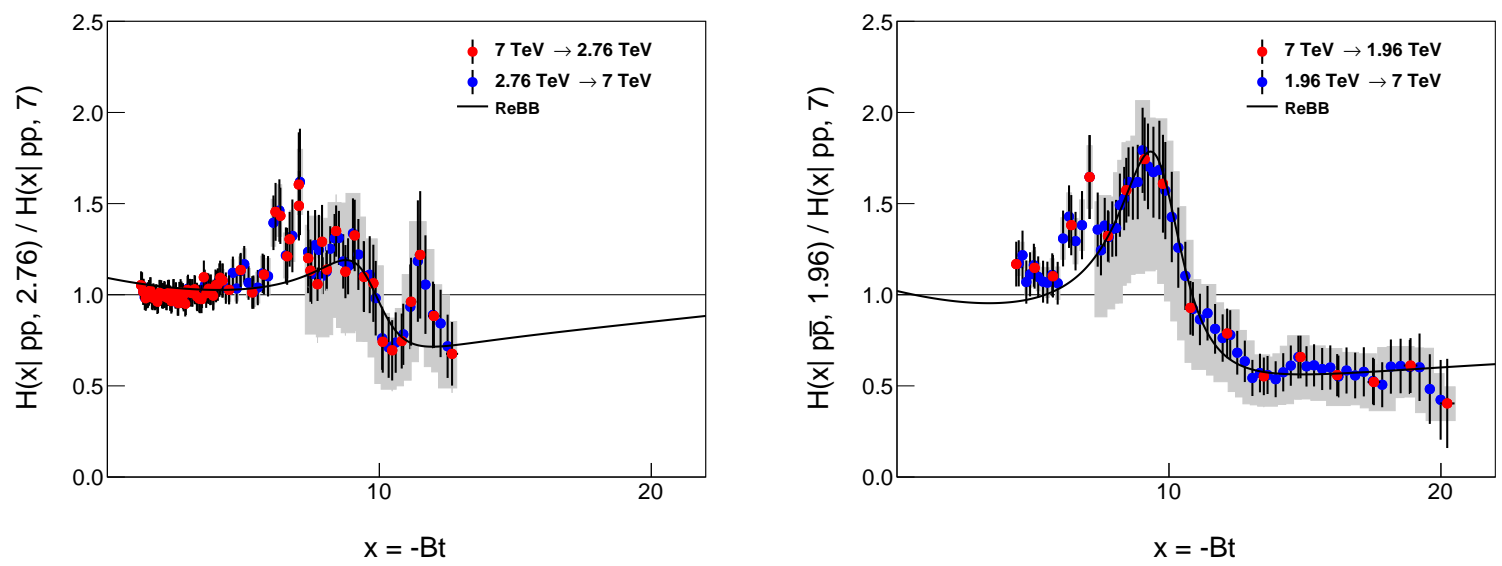

FIG. 2: Left panel indicates that for $p p$ elastic scattering the ratio of the scaling functions $H\left(x, s_{1}\right) / H\left(x, s_{2}\right)$, where $x=-t B, \sqrt{s_{1}}=2.76 \mathrm{TeV}$ and $\sqrt{s_{2}}=7 \mathrm{TeV}$, is not inconsistent with unity within statistical errors, due to the energy independence of the $H(x)$ scaling in the $2.76 \leq \sqrt{s_{1,2}} \leq 7$ $\mathrm{TeV}$ energy range. The right panel indicates a statistically significant deviation from unity of the ratios of the $H(x)$ scaling functions for elastic $p \bar{p}$ collisions at $\sqrt{s}=1.96 \mathrm{TeV}$, and that of $p p$ collisions at $\sqrt{s}=7 \mathrm{TeV}$. Notation and the experimental data are the same as in Fig. 1, but represented here as ratios. On both panels, curved solid lines indicate the results of Refs. [30 32] The straight solid black lines at unity correspond to the scaling limit in both panels.

Let us also demonstrate the presence of the Odderon in elastic scattering in the $\mathrm{TeV}$ energy range in a new, qualitative manner by representing the Odderon effect as a peak in deviation from the baseline of a background, normalized to unity. On both panels of Fig. 2 we plotted, on a linear scale, the ratio of two differential cross-sections that decrease over five orders of magnitude. This way of plotting follows the good practices of the field, established e.g. in Ref. [5]. This plot magnifies the possible differences and any trends in the deviations in a much more transparent way as compared to Fig. 1. In Fig. 2(left) we demonstrate that the ratio of the $H(x, s)$ scaling functions for elastic $p p$ scattering at two distinct energies of $\sqrt{s}=2.76$ and $7 \mathrm{TeV}$ is indeed not inconsistent with unity within statistical errors. This means that $H(x, s)$ with $x=-t B$ is energy-independent at least in the range of $2.76 \leq \sqrt{s} \leq 7 \mathrm{TeV}$ at $\mathrm{CL}=99 \%$. This also indicates that in the energy range of a few $\mathrm{TeV}$, the trivial energy dependence is indeed scaled out from the differential cross section of elastic $p p$ scattering in the $H(x, s) \simeq H(x)=\left(1 / B \sigma_{\text {el }}\right) d \sigma / d t$ function. As a cross-check of uncertainties, we have considered two distinct directions of projection: direct $2.76 \rightarrow 7 \mathrm{TeV}$ and inverse $7 \rightarrow 2.76 \mathrm{TeV}$ 
denoted by blue and red central points, respectively, and no significant difference has been observed. This shows remarkable stability of our results with respect to the details of the projection procedure. We have also observed the same picture for the ratio of the $H(x)$ scaling functions at $\sqrt{s_{1}}=7$ and $\sqrt{s_{2}}=8 \mathrm{TeV}, H\left(x, s_{1}\right) / H\left(x, s_{2}\right)$, for $p p$ collisions. A solid line is also added, to guide the eye and to indicate the magnitude of the scaling violations in this $p p$ to $p p$ comparison, estimated with the help of Refs. [30 32].

In Fig. 2 (right) we present visible and statistically significant deviation from unity in the ratio of the scaling functions of $p p$ and $p \bar{p}$ elastic scattering. The ratio of the $H(x)$ scaling functions is shown for elastic $p \bar{p}$ collisions at $\sqrt{s}=1.96 \mathrm{TeV}$ over that of $p p$ collisions at $\sqrt{s}=7$ $\mathrm{TeV}$. As a cross-check, we show the results of two different projection procedures: direct $1.96 \rightarrow 7 \mathrm{TeV}$ and inverse $7 \rightarrow 1.96 \mathrm{TeV}$ denoted by blue and red central points, respectively. No significant variation with respect to the direction of projection has been found. In both ways, we observe a statistically significant Odderon effect as a peak in the $5<x<10$ region, followed by a factor of two suppression or decrease from unity in a broad range of $10 \lesssim x=-t B \lesssim 20$. The statistical significance of the observed difference between the $p p$ and $p \bar{p}$ scaling functions has been found to be at least $6.26 \sigma$, consistently with the result of a direct comparison of the scaling functions as shown in Fig. 1. A solid line is added to this panel too in order to estimate the magnitude of the $H(x)$ scaling violations in this $p p$ to $p \bar{p}$ comparison [31, 32].

One may wonder if this statistically significant and model independent Odderon signal is due to an extrapolation procedure from $\sqrt{s}=7 \mathrm{TeV}$ down to $1.96 \mathrm{TeV}$, that assumes the validity of the $H(x)$ scaling. Figs. 16 and 17 in Ref. [32] address this question without any reference to the validity of the $H(x)$ scaling, but relying on the ReBB model of Ref. [31]. As these results include the terms that violate the $H(x)$ scaling, they allow not only for the evaluation of $p p d \sigma / d t$ down to $\sqrt{s}=1.96 \mathrm{TeV}$, but also for the extrapolation of $p \bar{p} d \sigma / d t$ up to $\sqrt{s}=2.76 \mathrm{TeV}$ as well as to higher energies. These validated extrapolations result in a $\chi^{2} / \mathrm{NDF}=24.28 / 13$ and $100.35 / 20$ at $\sqrt{s}=1.96$ and $2.76 \mathrm{TeV}$, respectively. Within the ReBB model, the combined Odderon significance of the $1.96 p \bar{p}$ and $2.76 \mathrm{TeV} p p$ data can be

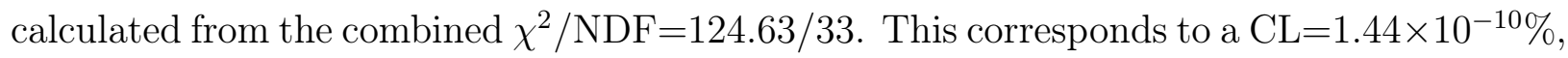
and to a model-dependent Odderon significance of $7.08 \sigma$. The same model [32] implies that the domain of validity of the $H(x, s)=H\left(x, s_{0}\right)$ scaling includes the $1.8 \leq \sqrt{s} \leq 8 \mathrm{TeV}$ energy range, where both $\rho(s)$ [7] and $\sigma_{e l}(s) / \sigma_{\text {tot }}(s)$ [34] are, within errors, independent of $s$. For 
some observables, this $s$-range may extend down to $\sqrt{s}=0.4 \mathrm{TeV}$ [32]. Our results can thus be cross-checked in elastic $p p$ collisions at $\sqrt{s}=510 \mathrm{GeV}$, with the STAR detector at the RHIC accelerator [42].

Our final, combined, conservative significance is an at least $6.26 \sigma$, crossing-odd effect in the scaling properties of $p p$ and $p \bar{p}$ scattering, obtained without any reference to modeling and without removing (or adding) any of the published D0 or TOTEM data points. If the high energy limit of proton is a black disk of gluons, elastic scattering becomes flavorblind and the leading squared logarithmic energy-dependent terms become the same for all reactions, from $p \pi$ to $p \gamma$ scattering [43]. Our result of a statistically significant Odderon observation implies, that elastic scattering is not flavor blind even at the $\mathrm{TeV}$ scale. Instead of the traditional black disc picture [43], our results thus support the newly emerging black ring picture of protons at asymptotically large energies [44].

Acknowledgments: We acknowledge inspiring and useful discussions with W. Guryn, G. Gustafson, V. A. Khoze, E. Levin, L. Lönnblad, M. Strikman, M. Sumbera and members of the D0 and TOTEM collaborations. R.P. is supported by the Swedish Research Council grants No. 621-2013-4287 and 2016-05996, by the European Research Council (ERC) under the European Union's Horizon 2020 research and innovation programme (grant agreement No 668679), as well as by the Ministry of Education, Youth and Sports of the Czech Republic project LTT17018. T. Cs., T. N., A. S. and I. Sz. were partially supported by the NKIFH grants No. FK-123842, FK-123959 and K-133046 as well as by the EFOP 3.6.1-16-201600001 grant (Hungary). Our collaboration has been supported by the COST Action CA15213 (THOR). 
* Electronic address: tcsorgo@cern.ch

$\dagger$ Electronic address: novak.tamas@szie.hu

$\ddagger$ Electronic address: Roman.Pasechnik@thep.lu.se

$\S$ Electronic address: ster.andras@wigner.hu

ฯ Electronic address: iszanyi@cern.ch

[1] L. Lukaszuk and B. Nicolescu, Lett. Nuovo Cim. 8, 405 (1973).

[2] J. Bartels, L. N. Lipatov, and G. P. Vacca, Phys. Lett. B477, 178 (2000), hep-ph/9912423.

[3] L. L. Jenkovszky, A. I. Lengyel, and D. I. Lontkovskyi, Int. J. Mod. Phys. A26, 4755 (2011), 1105.1202.

[4] A. Ster, L. Jenkovszky, and T. Csörgó, Phys. Rev. D91, 074018 (2015), 1501.03860.

[5] A. Breakstone, H. B. Crawley, G. M. Dallavalle, K. Doroba, D. Drijard, F. Fabbri, et al., Phys. Rev. Lett. 54, 2180 (1985).

[6] G. Antchev et al. (TOTEM), Eur. Phys. J. C79, 103 (2019), 1712.06153.

[7] G. Antchev et al. (TOTEM), Eur. Phys. J. C79, 785 (2019), 1812.04732.

[8] G. Antchev et al. (TOTEM), Eur. Phys. J. C79, 861 (2019), 1812.08283.

[9] G. Antchev et al. (TOTEM), Eur. Phys. J. C80, 91 (2020), 1812.08610.

[10] V. A. Khoze, A. D. Martin, and M. G. Ryskin, Phys. Rev. D97, 034019 (2018), 1712.00325.

[11] A. P. Samokhin and V. A. Petrov, Nucl. Phys. A974, 45 (2018), 1708.02879.

[12] T. Csörgő, R. Pasechnik, and A. Ster, Eur. Phys. J. C79, 62 (2019), 1807.02897.

[13] M. Broilo, E. G. S. Luna, and M. J. Menon, Phys. Rev. D98, 074006 (2018), 1807.10337.

[14] S. Pacetti, Y. Srivastava, and G. Pancheri, Phys. Rev. D99, 034014 (2019), 1811.00499.

[15] V. P. Gonçalves and P. V. R. G. Silva, Eur. Phys. J. C79, 237 (2019), 1811.12250.

[16] O. Selyugin and J. Cudell, Acta Phys. Polon. Supp. 12, 741 (2019), 1810.11538.

[17] V. A. Khoze, A. D. Martin, and M. G. Ryskin, Phys. Lett. B780, 352 (2018), 1801.07065.

[18] M. Broilo, E. G. S. Luna, and M. J. Menon, Phys. Lett. B781, 616 (2018), 1803.07167.

[19] S. M. Troshin and N. E. Tyurin, Mod. Phys. Lett. A33, 1850206 (2018), 1805.05161.

[20] I. M. Dremin, Universe 4, 65 (2018).

[21] E. Martynov and B. Nicolescu, Phys. Lett. B786, 207 (2018), 1804.10139.

[22] E. Martynov and B. Nicolescu, Eur. Phys. J. C 79, 461 (2019), 1808.08580. 
[23] Y. M. Shabelski and A. G. Shuvaev, Eur. Phys. J. C78, 497 (2018), 1802.02812.

[24] V. A. Khoze, A. D. Martin, and M. G. Ryskin, Phys. Lett. B784, 192 (2018), 1806.05970.

[25] Y. Hagiwara, Y. Hatta, R. Pasechnik, and J. Zhou (2020), 2003.03680.

[26] C. Contreras, E. Levin, R. Meneses, and M. Sanhueza (2020), 2004.04445.

[27] E. Gotsman, E. Levin, and I. Potashnikova (2020), 2003.09155.

[28] V. M. Abazov et al. (D0), Phys. Rev. D86, 012009 (2012), 1206.0687.

[29] G. Antchev et al. (TOTEM), EPL 101, 21002 (2013).

[30] A. Bialas and A. Bzdak, Acta Phys. Polon. B38, 159 (2007), hep-ph/0612038.

[31] F. Nemes, T. Csörgő, and M. Csanád, Int. J. Mod. Phys. A30, 1550076 (2015), 1505.01415.

[32] T. Csörgố and I. Szanyi (2020), 2005.14319.

[33] T. Csörgő, T. Novák, R. Pasechnik, A. Ster, and I. Szanyi (2019), 1912.11968v2.

[34] T. Csörgó (TOTEM), EPJ Web Conf. 206, 06004 (2019), 1903.06992.

[35] A. Adare et al. (PHENIX), Phys. Rev. C77, 064907 (2008), 0801.1665.

[36] J. Kaspar, EPJ Web of Conferences 172, 06005 (2018), URL https://doi.org/10.1051/ epjconf/201817206005.

[37] U. Amaldi and K. R. Schubert, Nucl. Phys. B166, 301 (1980).

[38] A. Breakstone et al. (AMES-BOLOGNA-CERN-DORTMUND-HEIDELBERG-WARSAW), Nucl. Phys. B248, 253 (1984).

[39] G. Antchev et al. (TOTEM), EPL 101, 21003 (2013).

[40] G. Antchev et al. (TOTEM), EPL 95, 41001 (2011), 1110.1385.

[41] F. J. Nemes, PoS DIS2017, 059 (2018).

[42] J. Adam et al. (STAR), Phys. Lett. B 808, 135663 (2020), 2003.12136.

[43] M. M. Block and F. Halzen, Phys. Rev. Lett. 107, 212002 (2011), 1109.2041.

[44] T. Csörgő, R. Pasechnik, and A. Ster, Eur. Phys. J. C80, 126 (2020), 1910.08817. 